\title{
Indicadores de alojamiento relacionados al bienestar animal en vacas lecheras
}

\author{
Alejos, J.I.; Almaraz, B.I.'; Peralta, J.G. ${ }^{2}$; Meza, N.M. ${ }^{2}$; Torres, M.G. ${ }^{2}$ \\ ${ }^{1}$ Univ. Autón. Chapingo, Depto. Zootecnia, Texcoco, México. ${ }^{2}$ Univ. Autón. \\ Hidalgo, Instit. Cs. Agropec. Área Acad. Med. Vet. \& Zoot. Hidalgo, México. \\ E-mail: maria_torres7599@uaeh.edu.mx
}

\begin{abstract}
Resumen
Alejos, J.I.; Almaraz, B.I.; Peralta, J.G.; Meza, N.M.; Torres, M.G.: Indicadores de alojamiento relacionados al bienestar animal en vacas lecheras. Rev. Vet. 32: 2, 174-180, 2021. El bienestar animal actualmente ha tomado auge en el mundo, sobre todo por sus repercusiones éticas, productivas y económicas. Los protocolos de evaluación para su estimación son una herramienta útil ya que permiten identificar los factores que disminuyen la calidad de vida de los animales, para estar en condiciones de mejorarla y hacer de la producción pecuaria una actividad cada vez más ética, humanitaria y eficiente. Por ello, el objetivo de esta investigación fue evaluar indicadores de bienestar animal asociados al alojamiento de vacas lecheras en unidades de producción a baja y mediana escala en la zona centro de México. Se evaluaron 2600 vacas lecheras utilizando parcialmente el protocolo propuesto por Welfare Quality ${ }^{\mathbb{Q}}$ : tiempo necesario para echarse; colisión al momento de echarse; vacas que descansan parcial o totalmente fuera de la zona de descanso; limpieza en flancos, ubres y patas traseras; presencia de cojeras en diferente grado; presencia de áreas sin pelo y presencia de lesión o inflamación. Los valores encontrados en la mayoría de los indicadores evaluados fueron inadecuados considerando los parámetros sugeridos en el protocolo utilizado. El alojamiento de las vacas evaluadas no brinda las condiciones necesarias para el descanso apropiado, por lo que se recomienda realizar acciones urgentes en la zona evaluada con la finalidad de mejorar la calidad del alojamiento y por ende del bienestar y productividad de los animales.
\end{abstract}

Palabras clave: alojamiento, bienestar animal, vacas lecheras.

\begin{abstract}
Alejos, J.I.; Almaraz, B.I.; Peralta, J.G.; Meza, N.M.; Torres, M.G.: Housing indicators related to animal welfare in dairy cows. Rev. Vet. 32: 2, 174-180, 2021. Animal welfare has now taken its rise in the world, especially due to its ethical, productive and economic repercussions. Animal welfare assessment protocols are a useful tool because they allow identifying the factors that reduce the quality of life of animals, to be able to improve it and make animal production an increasingly ethical, humane and efficient activity. Therefore, the aim of this research was to evaluate animal welfare indicators associated with housing of dairy cows of low and medium scale production units in central area of Mexico. 2600 dairy cows were evaluated using partially the protocol proposed by Welfare Quality $(\mathrm{B}$ : time needed to lie down; collision at the moment of lying down; cows that rest partially or totally outside there sting area; cleaning flanks, udders and hind legs; presence of lameness in different degree; presence of hairless areas and presence of injury or inflammation. The values found in most of the indicators evaluated were in adequate considering the parameters suggested in the protocol used. Housing cows evaluated, it does not provide the necessary for proper rest conditions, so it is recommended urgent action in the area to be assessed in order to improve the quality of housing and there fore the welfare and productivity of animals.
\end{abstract}

Key words: housing, animal welfare, dairy cows.

\section{INTRODUCCIÓN}

Históricamente el sector lechero ha enfrentado situaciones multifactoriales que afectan la productividad del hato, por lo cual se está constantemente en la búsqueda de herramientas que ayuden en la solución de dicha problemática. El bienestar animal es un tema que actualmente ha tomado auge en el mundo, dejando de ser una corriente meramente filosófica para convertirse en una ciencia con repercusiones éticas, productivas y económicas ${ }^{18,21,29}$. 
En los últimos años ha sido tema de investigación con el objetivo de desarrollar métodos para evaluarlo a nivel de granja y ofrecer al productor herramientas que le permitan aplicar estrategias que lo mejoren, aumentando en forma indirecta la productividad de los animales $^{2,26}$.

Se ha definido bienestar animal como "el estado de un individuo en sus intentos de mantenerse en equilibrio con su ambiente" "4 "cualquier punto de la calidad de vida que pueda tener influencia en el estado fisico o mental de un animal" ${ }^{32}$; se ha dicho también que un animal que vive con bienestar es aquel que está sano y tiene lo que quiere ${ }^{9}$

Para la Organización Mundial de Sanidad Animal (OIE), el bienestar animal designa "el estado físico y mental de un animal en relación con las condiciones en las que vive y muere" considerando que debe estar sano, cómodo, bien alimentado, que tiene la posibilidad de expresar formas innatas de comportamiento y que no sufre estados emocionales negativos como dolor, estrés, miedo y angustia, entre otros ${ }^{22}$

El concepto de bienestar animal implica el estado dinámico de un individuo en relación con los mecanismos biológicos que utiliza para adaptarse positiva y exitosamente ante los cambios del ambiente, involucrando salud, confort y estado emocional ${ }^{8}$.

Debido a la importancia y contribución del bienestar animal en la sanidad y la productividad del ganado, así como a la necesidad de reconocer su importancia en el comercio internacional, la OIE recomienda establecer a nivel mundial principios de bienestar animal en los procesos productivos, por lo que alienta el incremento de la investigación y la capacitación en esta temática.

En México, como en la mayoría de los países de América Latina, no se considera la evaluación del bienestar animal durante el proceso productivo de las unidades de producción de leche como una práctica rutinaria, pese a la evidencia científica que muestra su importancia en la productividad de los animales e inocuidad de la leche.

Para evaluar el bienestar de los animales es necesario considerar tres factores importantes: el funcionamiento biológico (salud, reproducción, productividad), la naturalidad (comportamiento) y el estado afectivo (etapas mentales) ${ }^{5,10,27}$. Al integrar estos tres factores en una estrategia de evaluación, se estará más cerca de evaluar el estado real del animal respecto a su bienestar, ya que el logro de uno sólo no garantiza que se logre un estado de bienestar ${ }^{27}$

El esfuerzo por desarrollar métodos para evaluar el bienestar animal en las unidades de producción ha crecido dramáticamente desde finales de la década de 1990, y puede evidenciarse con la publicación de diversos protocolos, entre los cuales destaca el desarrollado por el proyecto europeo Welfare Quality ${ }^{\circledR}$ para la evaluación en diversas especies ${ }^{1,17}$.

En el protocolo para la evaluación del bienestar en ganado lechero propuesto por la Welfare Quality ${ }^{\circledR}$, se
Tabla 1. Tiempo aproximado que las vacas dedican a diferentes actividades

\begin{tabular}{lc}
\hline actividad $^{13}$ & tiempo dedicado (h/día) \\
\hline comer & 3 a 5 h $(9$ a 14 visitas al comedero) \\
estar echada (descansar) & 12 a $14 \mathrm{~h}$ \\
interacciones sociales & $2 \mathrm{a} 3 \mathrm{~h}$ \\
rumiar & $7 \mathrm{a} 10 \mathrm{~h}$ \\
beber & $30 \mathrm{~min}$ \\
ordeñar (proceso completo) & $2.5 \mathrm{a} 3.5 \mathrm{~h}$ \\
\hline
\end{tabular}

incluyen 4 principios de evaluación del bienestar animal: buen alojamiento, buena alimentación, buena salud y comportamiento apropiado.

Respecto al bienestar de la vaca lechera, un punto clave es que ésta pueda permanecer descansando el mayor tiempo posible, por lo cual no debe impedirse o afectarse el número de horas que dedique para descansar. El descanso es una conducta que tiene relación directa con diferentes medidas del alojamiento, y se considera una conducta inelástica.

Por lo tanto, existe una necesidad biológica de alta motivación para realizarla; la imposibilidad de efectuarla le repercute al animal en serios problemas de salud, productividad, comportamiento y estados emocionales negativos.

La Tabla 1 muestra como la vaca divide las 24 horas del día para realizar diferentes actividades.

Tal como se muestra en la Tabla 1, la vaca dedica la mayor cantidad de tiempo para estar echada y descansar; sin embargo, una vaca que esté echada no necesariamente está descansando; el lugar de descanso, ya sea en cubículos (echaderos), asoleaderos o algún otro lugar, debe estar en condiciones adecuadas para que las vacas descansen confortable y relajadamente.

Las vacas tienen una alta motivación para descansar, y aunque prefieren hacerlo en un lugar confortable, si éste no existe, se echarán donde puedan aunque no estén cómodas, y por consiguiente, están en estrés continuo, lo cual resulta en una disminución del tiempo de descanso con varias repercusiones adversas para el bienestar de la vaca.

El descanso adecuado tiene diversos beneficios: menor estrés en las patas, lo que repercute en menor incidencia de cojeras; mayor flujo sanguínea hacia la ubre, lo que resulta en mayor producción de leche y mejor estado general del animal, lo cual genera como consecuencia una mayor eficiencia económica de la unidad de producción ${ }^{6}$.

Por lo anterior, el objetivo de esta investigación fue evaluar variables relacionadas con el alojamiento como indicador de bienestar en vacas lecheras en establos de mediana y baja producción.

\section{MATERIAL Y MÉTODOS}

\section{Localización}

Esta investigación se realizó en siete municipios del Valle de Tulancingo, ubicados en el Estado de Hidalgo, 
Tabla 2. Resumen de las variables evaluadas asociadas al alojamiento como indicador de bienestar animal.

\begin{tabular}{ll}
\hline grupo de variables & variables \\
\hline $\begin{array}{ll}\text { variables que evalúan } \\
\text { la conducta de reposo }\end{array}$ & $\begin{array}{l}\text { a) tiempo requerido para echarse } \\
\text { b) porcentaje de vacas que colisionan al momento de echarse } \\
\text { c) porcentaje de vacas que descansan parcial o totalmente fuera }\end{array}$ \\
\hline $\begin{array}{ll}\text { variables que evalúan } & \text { a) porcentaje de vacas sucias de las patas traseras } \\
\text { la limpieza corporal } & \text { b) porcentaje de vacas sucias de los flancos } \\
\text { c) porcentaje de vacas sucias de la ubre }\end{array}$ \\
$\begin{array}{ll}\text { variables que evalúan } & \text { a) porcentaje de vacas con diferente grado de cojera } \\
\text { el estado sanitario } & \text { b) porcentaje de vacas con áreas sin pelo } \\
& \text { c) porcentaje de vacas con lesiones o inflamación }\end{array}$ \\
\hline
\end{tabular}

2.2. Variables que evalúan la limpieza corporal con relación al alojamiento.

La limpieza se define como el grado de suciedad, y se considera como mínimo una capa de suciedad del tamaño de la palma de la mano o más de la mitad de la zona en cuestión para considerarla como sucia; las partes consideradas fueron cuartos traseros, flancos y ubres. Para realizar esta evaluación, se observó

República Mexicana. Se evaluaron aproximadamente 2600 vacas en producción, de la raza Holstein, en sistemas de producción a baja y mediana escala, utilizando parcialmente la metodología propuesta en el protocolo de la Welfare Quality ${ }^{\circledR}{ }^{31}$ para la evaluación del bienestar animal en ganado lechero.

\section{Variables de respuesta}

Un resumen de las variables estudiadas se presenta en la Tabla 2.

\subsection{Variables que evalúan la conducta de reposo con relación al alojamiento.}

a) Tiempo requerido para echarse. Para medir esta variable se consideraron todos los movimientos observables necesarios para echarse. El conteo inició cuando una articulación del carpo se inclina y baja, y terminó cuando el cuarto trasero del animal ha caído. El tiempo en segundos se registró individualmente, y cada vaca se clasificó en uno de tres rangos: tiempo menor a 5.2 segundos, considerado como el rango normal; tiempo entre 5.3 y 6.3 segundos, considerado como un problema moderado; y tiempo mayor a 6.3 segundos, considerado como un problema grave. Posteriormente se calculó el porcentaje de vacas en cada rango.

b) Porcentaje de vacas que colisionan al momento de echarse. Una colisión se define como el contacto que ocurre cuando cualquier parte del cuerpo de la vaca choca con alguna parte de la instalación al momento de echarse. Considerando lo anterior, se registró la colisión al momento de presentarse cuando la vaca se echa, calculando posteriormente el porcentaje de vacas que colisionaron.

c) Porcentaje de vacas que descansan parcial o totalmente fuera de la zona de descanso. Se cuantificó el número de vacas que estaban echadas y la cantidad de ellas que tenían su cuarto trasero en el borde del echadero o completamente fuera del mismo. En unidades de producción donde no existían cubículos o echaderos individuales, se consideraron características inadecuadas del piso donde las vacas se echaban, tales como pisos mojados, con piedras, y con exceso de excremento, entre otras. Posteriormente se calculó el porcentaje de animales echados parcial o totalmente fuera del echadero, o en pisos inadecuados. a cada animal por ambos lados y por detrás, calculando posteriormente:

a) porcentaje de vacas sucias de las patas traseras

b) porcentaje de vacas sucias de los flancos

c) porcentaje de vacas sucias de las ubres

\subsection{Variables que evalúan el estado sanitario con re- lación al alojamiento.}

a) porcentaje de vacas con diferente grado de cojera. Para evaluar los diferentes grados de cojera, se hizo caminar a la vaca a evaluar, en línea recta sobre una superficie nivelada y no resbaladiza; observándose por detrás y por ambos lados. La calificación fue individual, y cada vaca se clasificó como no coja (sincronización del paso y soporte de peso igual en los 4 miembros), coja (ritmo desigual) o muy coja (no soportar el peso en una extremidad). Posteriormente se calculó el porcentaje de vacas en cada grado de cojera.

b) porcentaje de vacas con áreas sin pelo. Se consideró área sin pelo al espacio de piel no dañada, con adelgazamiento de la capa y posible hiperqueratosis. Se consideraron las áreas sin pelo mayores a dos centímetros, ubicadas en cualquier parte del cuerpo. Se calculó el porcentaje de vacas que presentaron áreas del cuerpo con esta característica.

c) porcentaje de vacas con lesiones o inflamaciones. Se consideró lesión o inflamación de la piel dañada ya sea con costra o herida, dermatitis por ectoparásitos, falta total o parcial de pezones y lesión de la oreja debido al arrancamiento de arete. Se calculó el porcentaje de vacas que presentaron áreas del cuerpo con estas características. En la Tabla 3 se presenta la clasificación de los parámetros esperados de las variables asociadas al alojamiento, propuestos por Welfare Quality ${ }^{\circledR}{ }^{31}, y$ que fue la base para clasificar los resultados de esta investigación.

\subsection{Aspectos éticos.}

Las vacas lecheras consideradas en esta investigación, no fueron sometidas a ningún manejo extra al que recibían de manera rutinaria en la unidad de producción, que pudiera ocasionarles riesgo o sufrimiento, ya que la evaluación se basó exclusivamente en la observación, registro y análisis de datos, tal como se descri- 
Tabla 3. Parámetros de evaluación de variables asociadas al alojamiento como indicador de bienestar animal (adaptado de Welfare Quality ${ }^{31}$ ).

\begin{tabular}{lccc}
\hline \multirow{2}{*}{ característica evaluada } & \multicolumn{3}{c}{ clasificación } \\
\cline { 2 - 4 } tiempo necesario para echarse (seg.) & normal & problema moderado & problema serio \\
porcentaje de vacas parcial o totalmente fuera del echadero & $<5.20 \mathrm{~s}$ & 5.20 a $6.30 \mathrm{~s}$ & $>6.30 \mathrm{~s}$ \\
porcentaje de vacas que colisionan al momento de echarse & $<3 \%$ & $3 \%$ a $5 \%$ & $>5 \%$ \\
porcentaje de vacas con patas traseras sucias & $<20 \%$ & $20 \%$ a 30\% & $>30 \%$ \\
porcentaje de vacas con ubre sucia & $<20 \%$ & $20 \%$ a 50\% & $>50 \%$ \\
porcentaje de vacas con flancos sucios & $<10 \%$ & $10 \%$ a 19\% & $>19 \%$ \\
porcentaje de vacas algo cojas & & $10 \%$ a 19\% & $>19 \%$ \\
porcentaje de vacas muy cojas & & máximo aceptable $7 \%$ & \\
porcentaje de vacas con áreas sin pelo & máximo aceptable 3\% & \\
porcentaje de vacas con lesión o inflam. & & máximo aceptable $10 \%$ & \\
\hline
\end{tabular}

Tabla 4. Resultados de variables que evalúan conducta de descanso y limpieza corporal con relación al alojamiento como indicador de bienestar animal.

\begin{tabular}{lcc}
\hline característica evaluada & promedio encontrado & clasificacion $^{31}$ \\
\hline tiempo necesario para echarse $(\mathrm{s})$ & $<5.2 \mathrm{~S}, 26 \%$ de vacas & normal \\
& $5.2-6.3 \mathrm{~s}, 67 \%$ de vacas & problema moderado \\
problema serio \\
porcentaje de vacas parcial o totalmente fuera del echadero & $>6.30 \mathrm{~s}, 7 \%$ de vacas & problema grave \\
porcentaje de vacas que colisiona al momento de echarse & $100 \%$ & problema serio \\
porcentaje de vacas con patas traseras sucias & $30 \%$ & problema grave \\
porcentaje de vacas con ubre sucia & $97 \%$ & problema grave \\
porcentaje de vacas con flancos sucios & $76 \%$ & problema grave \\
\hline
\end{tabular}

be en el apartado variables de respuesta de "Material y Métodos"; por lo tanto, no se estimó necesario contar con el aval de un comité de ética y cuidado animal para la realización, dada la naturaleza exclusivamente observacional de esta investigación.

\section{RESULTADOS}

1. Variables que evalúan la conducta de descanso con relación al alojamiento como indicador de bienestar animal.

Respecto al tiempo necesario para "echarse", se encontró que $44.47 \%$ de las vacas lo hicieron en menos de 5.2 segundos, considerado como el tiempo promedio normal que una vaca necesita para realizar esa conducta. Sin embargo, el $38.89 \%$ de las vacas necesitaron entre 5.2 y 6.3 segundos, lo que se asocia con problemas moderados en la conducta de reposo; $16.64 \%$ de las vacas necesitaron más de 6.3 segundos, lo que se asocia con graves problemas en la conducta de reposo. Se encontró también que $100 \%$ de las vacas evaluadas se echaron total o parcialmente fuera del echadero en unidades de producción que cuentan con echaderos individuales, o en pisos inadecuados en aquellas que no contaban con echaderos individuales. Además, se encontró que el 30\% de las vacas que se echaron, colisionaron con alguna estructura que forma parte de las instalaciones como tubos o bardas (Tabla 4)

\section{Variables que evalúan la limpieza corporal con relación al alojamiento como indicador de bienestar animal.}

Respecto a la limpieza de las vacas, se encontró que el grado de suciedad en las tres áreas corporales que se evaluaron, constituye un problema grave, ya que el $97 \%$ de las vacas presentaron suciedad en las patas traseras, $76 \%$ suciedad en las ubres y $85 \%$ suciedad en los flancos (Tabla 4).

\section{Variables que evalúan el estado sanitario con re- lación al alojamiento como indicador de bienestar animal.}

Respecto al estado sanitario en lo referente al porcentaje de vacas con diferente grado de cojera, se encontró $4 \%$ de vacas muy cojas, mayor al porcentaje indicado en el protocolo como máximo aceptable (3\%); y $5 \%$ de vacas cojas, menor al mínimo aceptable (7\%). Se encontró que $42 \%$ y $16 \%$ de las vacas evaluadas presentaron áreas sin pelo y con lesiones y/o inflamación, respectivamente, resultados que constituyen un problema 
grave, ya que lo máximo aceptable según el protocolo utilizado es de $10 \%$ para cada característica evaluada (Tabla 5).

\section{DISCUSIÓN}

Los resultados encontrados en la evaluación de las variables que evalúan la conducta de descanso reflejan que los espacios destinados para esta conducta no son los apropiados, y evidencian diseños inadecuados y espacios limitados, donde predominan lugares mojados, con piedras y exceso de excrementos. Al no haber espacios suficientes y adecuados para echarse y descansar, el bienestar y la productividad de la vaca disminuyen.

La colisión de las vacas cuando se echan se interpreta como falta de comodidad y puede ocasionarles lesiones en diferentes partes del cuerpo, así como reducir el tiempo de descanso. La disminución en el lapso dedicado al descanso, ocasionado por espacios inadecuados, puede conducir por lo menos a dos de los principales problemas de bienestar en el ganado lechero.

En primer lugar, el riesgo de cojera aumenta si los animales no descansan lo suficiente, esto es particularmente importante en el ganado lechero. En segundo lugar, las vacas lecheras tienen una fuerte motivación para descansar, conducta que se considera inelástica si no lo hacen (Tabla 1), pudiendo experimentar estados emocionales negativos como la frustración y la angustia $^{7,11,20}$.

Cuando los espacios dedicados a descansar son inadecuados, los animales pueden presentar secuencias anormales de movimientos para echarse y levantarse, lo cual aumenta el riesgo de lesiones, dolor y angustia. Además, espacios de descanso insuficientes pueden ocasionar mayor competencia y mayor número de comportamientos agonistas.

Una de las funciones biológicas que ocurren en el largo período de descanso de las vacas lecheras en superficies confortables y cómodas (limpias, secas y suaves) es el aumento del flujo sanguíneo de la ubre y la consiguiente disponibilidad de nutrientes en la glándula mamaria, resultando en el incremento de la biosíntesis de leche, de tal modo que -si el tiempo dedicado al reposo se reduce- la producción de leche disminuirá.

En estudios previos se ha demostrado que en vacas echadas cómodamente, el flujo sanguíneo hacia la ubre aumenta entre 15 y $25 \%$ en comparación a una vaca que se encuentre de pie; y se estima que se incrementa aproximadamente en un kilo de leche producida al día por cada hora adicional de descanso ${ }^{28,30}$

Otro factor que se afecta cuando las vacas no descansan adecuadamente, es la disminución del consumo de materia seca. Se ha observado que cuando se las mantiene de pie (por malas condiciones de cubículos o área de descanso), reducen el tiempo dedicado para alimentarse hasta 30 minutos por cada hora adicional que estén de pie. Esto es particularmente importante considerando que el consumo voluntario de materia seca es uno de los principales factores que limitan la
Tabla 5. Resultados de variables que evalúan el estado sanitario con relación al alojamiento como indicador de bienestar animal ${ }^{31}$

\begin{tabular}{lcc}
\hline caracteristica evaluada & $\begin{array}{c}\text { \% máximo } \\
\text { recomendado o }\end{array}$ & $\begin{array}{c}\% \\
\text { hallado }\end{array}$ \\
\hline \% de vacas cojas & $7 \%$ & $5 \%$ \\
$\%$ de vacas muy cojas & $3 \%$ & $4 \%$ \\
$\%$ de vacas con áreas sin pelo & $10 \%$ & $42 \%$ \\
$\%$ con lesiones o inflamación & $10 \%$ & $16 \%$ \\
\hline
\end{tabular}

producción de las vacas lecheras, sobre todo en la etapa de la lactancia temprana ${ }^{14}$

La conducta de descanso es incluso más importante para las vacas lecheras que la conducta de alimentación y la interacción con otras vacas. El tiempo que las vacas dedican al descanso es importante, ya que la reducción del lapso que permanecen echadas puede afectar tanto a la producción como al bienestar. Cuando está echada, una vaca optimiza la rumia y produce más saliva, reduciendo así el riesgo de acidosis ruminal.

El tiempo de descanso también afecta al lapso de rumia. Se ha observado que los bovinos pasan significativamente más tiempo rumiando, estando echados que estando de pie; la rumia es indispensable y de suma importancia ya que gracias a ella se utiliza más eficientemente el alimento ${ }^{26}$

Asimismo, el aporte de sangre a la ubre es mayor en una vaca echada (alrededor de $5 \mathrm{~L} / \mathrm{min}$ ) que en una vaca de pie (alrededor de $3 \mathrm{~L} / \mathrm{min}$ ); esto mejora la función de la ubre y aumenta la producción de leche. Cuando una vaca está demasiado tiempo de pie, la presión dentro de la cápsula de la pezuña aumenta y ello produce hipoxia (reducción del suministro de oxígeno) e isquemia (flujo sanguíneo restringido), aumentando el riesgo de cojeras.

Por otra parte, la competencia por un lugar cómodo para descansar puede desencadenar conflictos entre las vacas, lo cual a su vez puede causar estrés crónico y aumentar el riesgo de enfermedades y problemas reproductivos. El alojamiento tiene un impacto sustancial sobre la conducta de descanso, la salud y el rendimiento de las vacas lecheras ${ }^{7}$.

La limpieza de los animales es un aspecto muy importante que se debe considerar como indicador de bienestar animal. Las vacas mantienen generalmente su pelaje limpio, excepto los cascos que pueden ensuciarse al caminar por terrenos con lodo. Un pelaje sucio indica que el animal es mantenido en condiciones precarias de higiene ${ }^{16}$

La falta de limpieza corporal es evidencia de que las vacas se echan en espacios inadecuados, ya sea en pisos mojados o con exceso de excremento, y la presencia de suciedad en las áreas evaluadas es un factor de riesgo importante ${ }^{23}$.

Altos niveles de suciedad en las piernas y flancos se asocian a mayor riesgo de cojera, dermatitis digital y dermatitis interdigital; alto nivel de suciedad en ubre se asocia a mayor incidencia de mastitis ${ }^{24,25}$, ya que 
se incrementa la exposición a patógenos ambientales como E. coli y Streptococcus uberi ${ }^{3}$. La mastitis es uno de los principales problemas que disminuyen considerablemente el bienestar de vacas lecheras, ya que ocasiona dolor intenso y sufrimiento, además de sus repercusiones en la productividad y calidad de la leche ${ }^{23}$.

Las afecciones podales se consideran como uno de los factores más importantes que disminuyen el bienestar de las vacas lecheras, sólo superado por problemas reproductivos y mastitis, pues se relacionan con el dolor (experiencia emocional aversiva que sufren los animales) y alteración de su comportamiento natural, por lo cual es muy importante considerarlo en la evaluación del bienestar 12, 14, 29, 33 .

La presencia de cojeras limita las conductas de desplazamiento y búsqueda, lo cual repercute en la disminución de consumo voluntario de alimento y agua; así como por su repercusión en la función locomotora y por el agobio de la vaca por el dolor producido, que merma la capacidad productiva, disminuye la condición corporal y la fertilidad, incluso induce al envejecimiento precoz ${ }^{15,19,27}$ lo cual eventualmente incrementa la tasa de reemplazo del hato. Las cojeras en vacas son condiciones extremadamente dolorosas y se deben tomar urgentes medidas para reducir su incidencia.

Debe tenerse en cuenta que las lesiones podales o cojeras tienen un origen multifactorial, por lo que en su prevención deben tenerse en cuenta las características del suelo (evitar suelos duros, húmedos y resbaladizos), densidad animal, espacios de comederos, número y diseño de cubículos individuales de descanso, áreas de sombra, cambios frecuentes en la composición social del grupo, diseño de pasillos, manejo brusco de los animales, estrés calórico, cambios bruscos en la alimentación, cuidado inadecuado de pezuñas y -en general- prácticas que obliguen a las vacas a permanecer demasiado tiempo de pie ${ }^{6}$

Las lesiones corporales pueden ser también indicadoras de instalaciones mal diseñadas o insuficientes. El hecho que los animales descansen en lugares inapropiados; pueden causar dolor agudo o crónico, y es -por lo tanto- un problema de bienestar. Los flancos y las patas son las partes del cuerpo que se lesionan con mayor frecuencia en animales de granja.

Tales lesiones interfieren con el comportamiento normal y la locomoción, pudiendo tener efecto debilitante mediante la privación de la alimentación. Las heridas pueden infectarse y -en algunas circunstanciaspueden ocasionar enfermedad sistémica. Además, las infecciones, las enfermedades sistémicas secundarias a las lesiones, así como el efecto debilitante de algunas lesiones, pueden ocasionar que los animales sean sacrificados de manera precoz ${ }^{14,33}$

La ubicación de lesiones e inflamaciones o áreas sin pelo pueden servir para ubicar el origen del problema; las encontradas en piernas, rodillas o corvejones se asocian a superficies de descanso abrasivas, insuficientes y duras, mientras que las ubicadas en el cuello indican comederos mal diseñados.
El alto porcentaje de vacas muy cojas, vacas con áreas sin pelo y vacas con lesiones e inflamaciones encontradas en esta investigación, son consecuencias de instalaciones inadecuadas que no permiten a la vaca realizar sus actividades diarias de manera normal (cuidado corporal, descanso, interacciones sociales, ejercicio), causando daños corporales que finalmente repercuten en la disminución de su bienestar.

En conclusión, el gran porcentaje de vacas que necesitan tiempo excesivo para echarse, que colisionan al momento de echarse, que descansan en espacios inadecuados, que poseen suciedad en patas traseras, flancos y ubres, que presentan cojera grave así como áreas sin pelo o con lesiones o inflamaciones encontradas en este estudio, evidencian problemas graves de falta de bienestar en las vacas lecheras evaluadas.

Tales problemas son ocasionados principalmente por condiciones inadecuadas de alojamiento, lo cual podría repercutir en la alteración de la conducta de descanso y por consiguiente del nivel productivo, además de ocasionar incomodidad, dolor, frustración y sufrimiento en los animales.

Monitorear periódicamente los indicadores de bienestar asociados al alojamiento de manera objetiva, permitirá al productor implementar estrategias que le admitan mejorar las condiciones de alojamiento de las vacas, y -por ende- incrementar su bienestar y productividad, lo cual eventualmente repercutirá no solo en el aumento del bienestar de las vacas, sino también en una mayor eficiencia productiva de la unidad de producción.

\section{REFERENCIAS}

1. Andreasen SN. 2013. The correlation of qualitative benhavior assessments whit welfare quality protocol in onfarm welfare assessment of dairy cattle. Appl Anim Behav Sci 143: 9-17.

2. Arraño C, Baez A, Flor E, Whay HR, Tadich N. 2007 Estudio preliminar del uso de un protocolo para evaluar el bienestar de vacas lecheras usando observaciones basadas en el animal. Arch MedVet 39: 239-245.

3. Bradley AJ, Green M. 2000. A study of the incidence and significance of intra-mammary entero-bacterial infection acquired during the dry period. J Dairy Sci 83: 1957-1965.

4. Broom DM. 2008. Welfare assessment and relevant ethical decisions: key concepts. Ann Rev Biomed Sci 10: T79T90.

5. Broom DM. 2010. Animal welfare: an aspect of care, sustainability and food quality required by the public. Journ Vet Med Educat 37: 83-88.

6. Callejo RA. 2009. Cow comfort: el bienestar de la vaca lechera, Editorial Servet, Zaragoza, España.

7. Cook NB, Nordlund KV. 2009. The influence of the environment on dairy cow behavior, claw health and herd lameness dynamics. Vet Journal 179: 360-369.

8. Corrado MV. 2009. Animal welfare: review of the scientific concept and definition. Italian Journ of Anim Scien 8: $1,21-30$. 
9. Dawkins MS. 2017. Animal welfare and efficient farming is conflict inevitable? Anim Product Sci 57: 201-208

10. Duncan IJ, Fraser D. 1997. Understanding animal welfare. In: M.C. Appleby \& B.O. Hughes (Eds.), Animal Welfare, UK: CAB Intl URL.

11. Endres MI, Barberg AE. 2007. Behavior of dairy cows in an alternative bedded pack housing system. J Dairy Sci 90: 4192-4200.

12. Galindo F, Broom D. 2000. The relationships between social behavior of dairy cows and the occurrence of lameness in three herds. Res Vet Sci 69: 1, 75-79.

13. Grant R.J. 2006. Incorporating dairy cow behavior into management tools. Penn State Dairy Cattle Nutrition Workshop, Pp. 31-41, URL.

14. Green LE, Borkert J, Monti G, Tadich N. 2010. Associations between lesion-specific lameness and the milk yield of 1635 dairy cows from seven herds in the $\mathrm{X}$ region of Chile and implications for management of lame cows world wide. Anim Welf 19: 419-427.

15. Horseman SV et al. 2014. The use of in-depth interviews to understand the process of treating lame dairy cows from the farmers' perspective. Anim Welf 23: 157-165.

16. Hughes J. 2001. A system for assessing cow cleanliness. In Practice 23: 517-524.

17. Keeling L. 2009. An overview of the development of the welfare quality project. Assessment systems, W.Q. reports 12, Cardiff University, United Kingdom.

18. McInerney JP. 2004. Animal welfare, economics and policy. Report on a study undertaken for the Farm \& Animal Health Economics Division of Defra. URL.

19. Miguel GG et al. 2014. Behavioural changes in dairy cows with lameness in an automatic milking system. Appl Anim Behav Sci 150: 1-8

20. Munksgaard L et al. 2005. Quantifying behavioural priorities. Effects of time constraints on behaviour of dairy cows, Bos taurus. Appl Anim Behav Sci 92: 3-14.

21. OIE. 2004. Global conference on animal welfare: an OIE initiative. World Org for Anim Health, OIE. Paris, France, $333 \mathrm{p}$.
22. OIE. 2008. Bienestar Animal, París: Organización Mundial de Sanidad Animal, Boletín 2: 69 pp.

23. Reneau JK et al. 2005. Association between hygiene scores and somatic cell scores in dairy cattle. $J$ Am Vet Med Assoc 227: 8, 1297-1301.

24. Santanna AC, Paranhos JR. 2011. The relationship between dairy cow hygiene and somatic cell count in milk. $J$ Dairy Sci 94: 3835-3844.

25. Schreiner DA, Ruegg PL. 2003. Relationship between udder and leg hygiene scores and subclinical mastitis. $J$ Dairy Sci 86: 11, 3460-3465.

26. Tadich N. 2011. Bienestar animal en bovinos lecheros Rev Colomb Cienc Pec 24: 293-300.

27. Vonkeyserlingk MA, Rushen J, Pasille AM, Weary DM. 2009. The welfare of dairy cattle. Key concepts and the role of science. J Dairy Sci 94: 4101-4111.

28. Vries $\mathbf{M}$ et al. 2011. Invited review: associations between variables of routine herd data and dairy cattle welfare indicators. J Dairy Sci 94: 7, 3213-3228.

29. Warnick LD, Janssen D, Guard CL, Grohn YT. 2001 The effect of lameness on milk production in dairy cows. J Dairy Sci 84: 9, 1988-1997.

30. Webster J. 2005. The assessment and implementation of animal welfare: theory into practice. Rev $S_{c i}$ Tech Off Int Epizoot 24: 2, 723-734.

31. Welfare Quality. 2011. Welfare Quality ${ }^{\circledR}$ assessement protocol for cattle. Consortium, Lelystad, Netherlands, npp 215. http://www.welfarequality.net.

32. Whay HR, Main DC, Green L, Webster AF. 2003. Animal based measures for the assessment of welfare state of dairy cattle, pigs, laing hens: consensus of expert opinion. Animal Welfare 12: 205-217.

33. Zimmerman A. 2001. Lameness in dairy cattle: are activity levels, hoof lesions and lameness correlated ? Thesis Master of Science, University of British Columbia, Canada. https://scielo.conicyt.cl/scielo.php 